\title{
Searching for self-enrichment in Cygnus OB2
}

\author{
Sara R. Berlanas ${ }^{1,2}$, Artemio Herrero ${ }^{1,2}$, Fernando Comerón ${ }^{3}$, \\ Anna Pasquali ${ }^{4}$ and Sergio Simón-Díaz ${ }^{1,2}$ \\ ${ }^{1}$ Instituto de Astrofísica de Canarias, 38205 La Laguna, Tenerife, Spain \\ ${ }^{2}$ Departamento de Astrofísica, Universidad de La Laguna, 38206 La Laguna, Tenerife, Spain \\ ${ }^{3}$ ESO, Karl-Schwarzschild-Strasse 2, 85748 Garching bei München, Germany \\ ${ }^{4}$ Astronomisches Rechen-Institut, Zentrum fr Astronomie der Universität Heidelberg, \\ Mönchhofstr 1214, 69120 Heidelberg, Germany
}

Cygnus OB2 is a rich and relatively close $(\mathrm{d} \sim 1.4 \mathrm{kpc})$ OB association in our Galaxy. It represents an ideal testbed for our theories about self-enrichment processes produced by pollution of the interstellar medium by successive generations of massive stars. Comerón \& Pasquali (2012, A\&A, 543, A101) found a correlation between the age of young stellar groups in Cygnus OB2 and their Galactic longitude. If is associated with a chemical composition gradient, it could support these self-enrichment processes.

In this work we have checked whether there is a correlation between abundances (as derived from OB-type stars) and star location in Cygnus OB2. For this purpose, we have performed a spectroscopic analysis of $7 \mathrm{OB}$ stars with low vsini in the association that a) are suitable for the spectroscopic analysis and b) cover the positions of all the age groups in the area. We have used FASTWIND stellar atmosphere models to determine their fundamental parameters as well as the Si and O surface abundances.

We have carried out an analysis based on equivalent widths (EW) of metal lines, similar to the method used in Orion by Simón-Díaz (2010, A\&A, 510, A22). The stellar parameters (Teff and logg) have been obtained from observed $\mathrm{H}$ Balmer lines and the EW ratios of Si II-III-IV. Then, we have applied the curve-of-growth method to derive the associated $\mathrm{Si}$ and $\mathrm{O}$ abundances.

Our abundance analysis indicates a potential correlation between $\mathrm{O}$ abundances and Galactic longitudes (see Fig.1), which we do not find for Si. We derive a total variation of the $\mathrm{O}$ abundance of about 0.3-0.5 dex, larger than intrinsic uncertainties. This correlation, together with the one found by Comerón \& Pasquali (2012), implies a possible evidence of self-enrichment in the region, taking into account that Cyg OB2 has an extended starformation history sufficiently long to have included the explosion of its oldest massive members as core-collapse supernovae. Therefore, the young generations of stars could have been enriched with the products from supernovae ejecta from older subgroups.

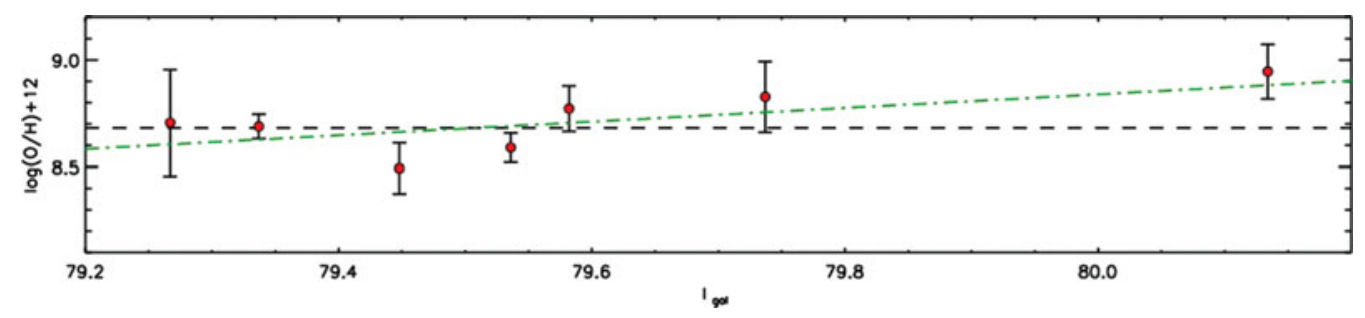

Figure 1. Derived O abundances vs Galactic longitude of our sample of OB stars with low vsini. Black dashed line represents the mean abundance value, which is used as a reference to correlate the abundances. Dash-dotted green line represents the fit to the data. 